\title{
Resonant-type inertial impact motor with rectangular pulse drive
}

\author{
Yuting $\mathrm{Ma}^{1,2}$, Husain Shekhani ${ }^{2}$, Xintao Yan ${ }^{1}$, Minkyu Choi ${ }^{2}$ and Kenji Uchino ${ }^{2}$
}

1) CAS Key Lab of Bio-Medical Diagnostics, Suzhou Institute of Biomedical Engineering and Technology, Chinese Academy of Sciences, Suzhou, Jiangsu, 215163, PR China

2) International Center for Actuators and Transducers, The Pennsylvania State University, State College, PA, 16802, USA

Abstract: In this paper, a resonant-type piezoelectric inertial motor driven by rectangular pulse is developed. The rectangular pulse drive methodology is explained and its influence on output displacement of the stator is discussed in relation to four factors: frequency ratio, duty ratio, vibration amplitude ratio, and phase difference. A bending stator with second harmonics at its double fundamental resonant frequency is designed to generate saw-tooth type displacements under a rectangular pulse. It is composed of an aluminum plate $(20 \mathrm{~mm} \times 4 \mathrm{~mm} \times 2 \mathrm{~mm})$ sandwiched between two piezoelectric plates $(10 \mathrm{~mm} \times 4 \mathrm{~mm} \times 0.73 \mathrm{~mm})$. Experiments show that with optimized duty ratios, both the rotation speed and stall torque can be enhanced. The rotation direction of the rotor can be reversed by changing the duty ratio. The maximum no-load angular speed reaches 300rpm with driving voltage of $60 \mathrm{Vpp}$ at the fundamental resonant frequency of $35.3 \mathrm{kHz}$ in both directions. Using a preload of $1.6 \mathrm{~N}$, stall torque reaches $1220 \mu \mathrm{Nm}$ driven at $80 \mathrm{Vpp}$ and $35.3 \mathrm{kHz}$. The maximum power density and efficiency of the motor are $4.2 \mu \mathrm{W} / \mathrm{mm}^{3}$ and $2.1 \%$, respectively, 
which are higher than the previously reported piezoelectric inertial rotary motors. The proposed inertial motor features simple mechanical and electrical design, which makes it ideal for miniature electro-mechanical device applications.

Keywords: piezoelectric inertia motor, rectangular pulse drive, resonant-type, higher harmonics

\section{Introduction}

There is a strong demand for compact and high-power motors to meet recent requirements of miniaturized electronic devices, ranging from consumer electronics to medical equipment. Piezoelectric inertia motors, also known as "stickslip drive", usually have a simpler configuration than conventional ultrasonic motors, which allows for lower production cost and further miniaturization. These motors make use of the inertia of a mass to drive a rotor or slider to move in increments via friction contact, utilizing saw-tooth type displacement to have stick and slip phases between the stator and rotor.

Piezoelectric inertial motors have gained wide popularity since they were originally developed in the mid-1980s for fine positioning applications [1-3]. Other successful applications include micro robot [4], camera module [5-7], and zoom lens system [8]. The specifications of a piezoelectric inertia motor contain angular speed (linear speed), stall

torque (thrust), power density, and efficiency. Basically, a tradeoff between angular speed (linear speed) and stall torque (thrust) should be considered depending on different applications. Power density and efficiency are always expected to be high for fitting into micro electronic devices and reducing energy waste. 
Saw-tooth type voltage signal is usually used to drive a piezoelectric inertia motor. When the applied voltage increases at relatively low speeds, the rotor moves with the stator; when the applied voltage decreases quickly, the stator goes back to initial position and a slippage is generated between the stator and rotor. Accumulate movement of the rotor in one direction can be obtained by cyclic excitation. In addition to "stick-slip drive", the "slip-slip drive" is also developed based on the same principle. By changing the shape of the saw-tooth, the moving direction of the rotor can be reversed. We demonstrated clearly that the piezoelectric inertia motor driven by a saw-tooth type voltage signal can only work at off-resonance, because the saw-tooth type voltage produces sinusoidal type displacement at resonance due to frequency filtering effect [9]. Off-resonant drive suffers from low vibration amplitude and low frequency, which deteriorates the performance of the motor. Some motors can work at an ultrasonic frequency by reducing size, but still they are not driven at its resonance [6, 7]. However, resonant-type piezoelectric inertia motors can be constructed by using other driving mechanisms, which is a combination of two eigen-modes of the stator using two driving signals. The ratio of two driving frequencies, which correspond to high harmonics and fundamental resonant frequencies, is set to be $2: 1$.

Researchers have used various ways to construct the resonant-type piezoelectric inertia motors, for example, by combining the first and second resonant longitudinal modes [10], the first and third resonant longitudinal modes $[11,12]$, and the first and second resonant bending modes of a bending actuator with one end clamped [13]. In resonanttype piezoelectric inertia motors, performance of the actuator can be greatly enhanced by choosing material with high mechanical quality factor. However, in all the above mentioned resonant type piezoelectric inertia motors, the amplitude and phase difference of the two driving signals should be specially designed and coordinated, which 
increases the driving circuit complexity and cost. Our Penn State group, Tuncdemir et al. proposed a rectangular pulse drive to obtain saw-tooth type displacement of the piezo-actuator at its fundamental resonance [9]. Because the motor is driven with a simple single signal, the driver/power supply cost can be greatly reduced, compared with other motors discussed above.

The goal of this work is to provide a comprehensive explanation on the rectangular pulse drive and extend this drive to a piezoelectric inertia motor with a bending actuator. Previously, longitudinal and torsional modes were used [9], while the bending actuator exhibits large displacement. Besides, their resonant frequency can be tuned using thickness and length, versus the longitudinal mode presented in Ref.[9] whose resonant frequency only depends on the length of the actuator. This increases the resonant frequency tunability, which will allow this kind of motor to potentially meet a wide variety of performance and geometry specifications. In Section 2, analytical approach on the rectangular pulse is presented with four parameters, namely, frequency ratio, duty ratio, vibration amplitude ratio, and phase difference, being discussed. Thus, the design rule of the stator is obtained. In Section 3, a prototype was fabricated and characteristics of both the stator and motor were tested. Two kinds of rotors were used to evaluate the angular speed, stall torque, and load performances of the prototype motor. Power density and efficiency were calculated and a comparison with previously reported inertia rotary motors were made in the last section.

\section{Design of the resonant type inertial motor}

\subsection{Driving principle of the motor}


For an inertial impact motor, asymmetric motion of the stator (normally saw-tooth type displacement) is essential to produce the stick and slip motions with its friction partners. Since pure fundamental resonance can only produce sinusoidal vibration, contribution of higher order harmonics should be considered in order to produce saw-tooth type displacement of the stator at resonance. The rectangular pulse waveform generator, one of the easiest and simplest electric circuits, is a promising driving method with its functionality to contain multiple orders of frequency components. It can be written in Fourier functions as following:

$V(t, D)=\sum_{n=1}^{\infty}\left[a_{n} \cos (n \omega t)+b_{n} \sin (n \omega t)\right]$

$a_{n}=\frac{2 A}{n \pi} \sin (2 n \pi D)$

$b_{n}=\frac{2 A}{n \pi}[1-\cos (2 n \pi D)]$

where $V$ is the driving voltage, $A$ is the voltage amplitude, $\omega$ is the fundamental resonant frequency, $D$ is the duty ratio of the pulse waveform. The output displacement of the stator driven by pulse waveform is determined by four factors: duty ratio of the pulse waveform $(D)$, ratio of resonance frequencies $(n)$, ratio of vibration amplitude $\left(\mathrm{Q}_{\mathrm{r}}\right)$, and vibration phase difference $\left(\varphi_{e}\right)$ of its fundamental and higher harmonic mode. Qr is ratio of vibration amplitude of fundamental and higher harmonic mode with the same driving voltage. In the following analysis, only the harmonic mode closest to the fundamental mode (usually the second or third harmonic mode) is considered as the higher order harmonics are often highly damped and their influence on the final output is insignificant. It can be seen from the equation that 
contribution from the fundamental and higher harmonic mode can be tuned by changing the duty ratio $D$, without using two power supplies for providing the necessary magnitudes of the fundamental and higher harmonic mode.

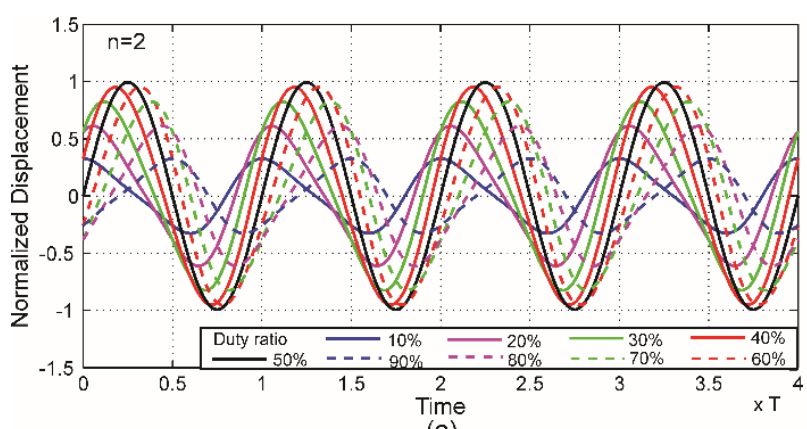

(a)

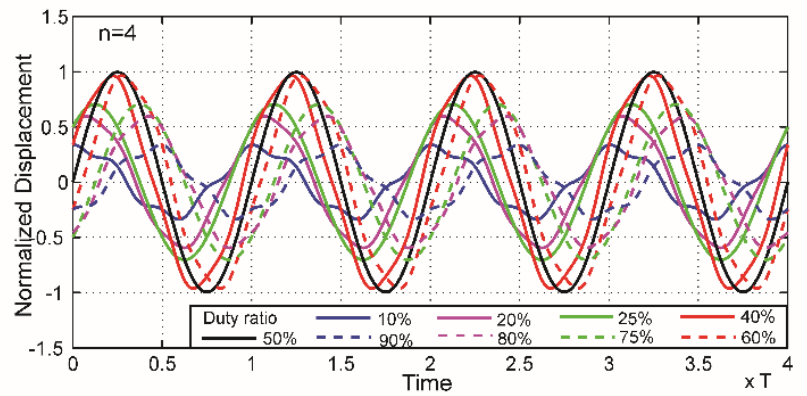

(c)

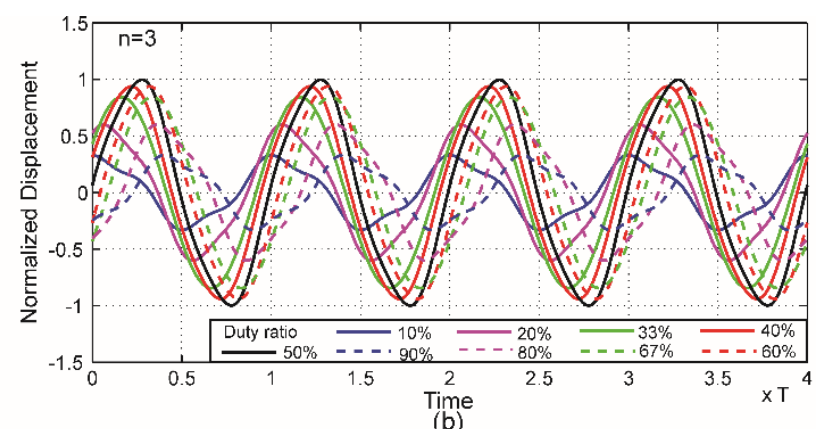

(b)

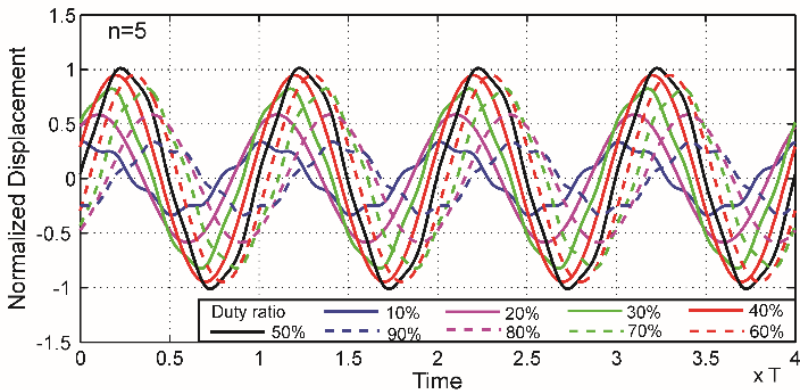

(d)

Fig.1 Normalized displacement of stator with high order harmonics at frequency of $n \times \omega(n=2,3,4,5)$ driven by rectangular pulse waveform and $\mathrm{Qr}=0.2, \varphi_{\mathrm{e}}=\pi / 2$.

Supposing that vibration amplitude of the stator is proportional to driving voltage amplitude at resonance, four cycles of combined displacement with high order harmonics at specific frequencies of $n \times \omega(n=2,3,4,5)$ is shown in Fig.1. Qr is set to 0.2 as the vibration amplitude of higher harmonics is usually much less than that of fundamental resonance and $\varphi_{\mathrm{e}}$ is fixed at $\pi / 2$. Fig.1(a) and (c) show that when $n=2$ or 4 , saw-tooth type displacement of the stator can be obtained by adjusting the duty ratio of pulse waveform. When the duty ratio is $50 \%$, only sinusoidal displacement is obtained. As the duty ratio deviates from $50 \%$, the displacement curve becomes more asymmetric and 
the vibration amplitude decreases. The moving direction of rotor can be reversed by changing the duty ratio from $D$ to 1-D. In Fig.1(b) and (d) where $n=3$ or 5 , saw-tooth type displacement cannot be generated although there are distortions on the displacement curve. It should also be noted that with duty ratio of $1 / n$ and $1-1 / n$, sinusoidal displacement curve is observed in Fig.1. Overall, when $n$ is even number, saw-tooth type displacement can be obtained except the duty ratio of $50 \%, 1 / n$ and $1-1 / n$. When $n$ is odd number, saw-tooth type displacement cannot be expected, because the velocity will cancel out during one rising and falling cycle.

Normalized displacement of the stator with different vibration amplitude ratio $\mathrm{Qr}$ is shown in Fig.2. The displacement is calculated by combining the fundamental and high order harmonics at frequency of $2 \omega$. The duty ratio of pulse waveform is set to 0.3 and $\varphi_{\mathrm{e}}$ is fixed at $\pi / 2$. As Qr increases, both saw-tooth type displacement asymmetry and vibration amplitude increase. Therefore, high order harmonics with large vibration amplitude can benefit the formation of saw-tooth type displacement. Three cases should be clarified: 1) For a high order harmonic at frequency of $n \times \omega$ where $n>5$, its driving amplitude highly reduces since $\mathrm{a}_{\mathrm{n}}$ and $\mathrm{b}_{\mathrm{n}}$ are proportional to $1 / n$, which barely influences final output. 2) If the ratio of vibration amplitude in the high order harmonic to the one in the fundamental resonance is very low, the high order harmonics can also be neglected. 3) If the frequency of high order harmonics deviates from $n \times \omega$, the integral multiple of fundamental resonant frequency, the contribution of the mode can be determined by the amplitude magnification coefficient $\beta_{\mathrm{i}}$,

$$
\beta_{i}=\frac{1}{\sqrt{\left(1-\lambda_{i}^{2}\right)^{2}+\left(2 \zeta_{i} \lambda_{i}\right)^{2}}} .
$$


where $\zeta_{\mathrm{i}}$ is the damping coefficient at high order resonant mode, $\lambda_{\mathrm{i}}$ is the frequency ratio of the nearest integral frequency to the high harmonic frequency. Therefore, when the high order harmonic mode is located at exact integral multiplied fundamental frequency, large vibration amplitude and the highest contribution on total output can be realized. Besides, piezoelectric materials with higher mechanical quality factor will also enhance performance of the stator with less damping of the vibration amplitude at the high order harmonics.

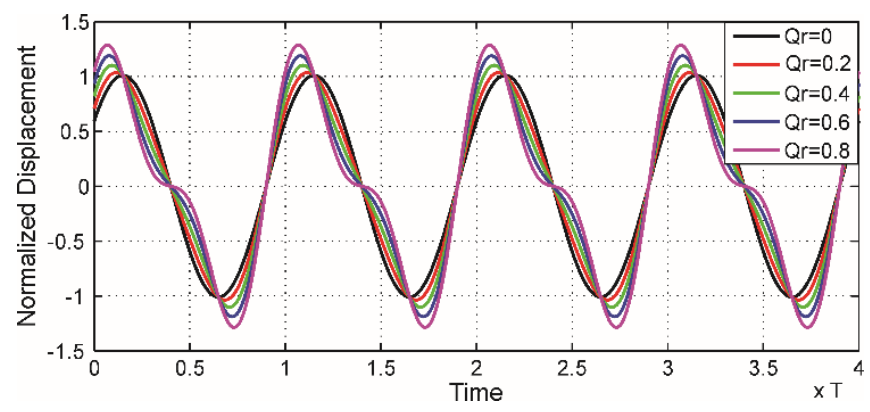

Fig.2 Normalized displacement of stator depending on vibration amplitude ratio. High order harmonics at $2 \omega$ is integrated with fixed $D=0.3$ and $\varphi_{\mathrm{e}}=\pi / 2$.

Normalized displacement of the stator depending on vibration phase difference $\varphi_{\mathrm{e}}$ is shown in Fig.3. The phase difference is defined as the time delay between two displacement curves over cycle of high order harmonic resonant displacement. The displacement is calculated by combining fundamental and $2 \omega$ harmonics. The duty ratio of the pulse waveform is set to 0.3 and $\mathrm{Qr}$ is fixed at $\pi / 2$. When the phase difference is integer multiple $\pi$, symmetric displacement is generated. As the phase difference varies in between $2 \mathrm{i} \pi$ to $(2 \mathrm{i}+1) \pi(\mathrm{i}=1,2,3 \ldots)$, the saw-tooth type displacement becomes asymmetric biased to left side. On the contrary, the displacement curve gets biased to right side 
when the phase difference is between $(2 \mathrm{i}+1) \pi$ and $(2 \mathrm{i}+2) \pi$. Therefore, the moving direction of the rotor can be influenced by the phase difference.
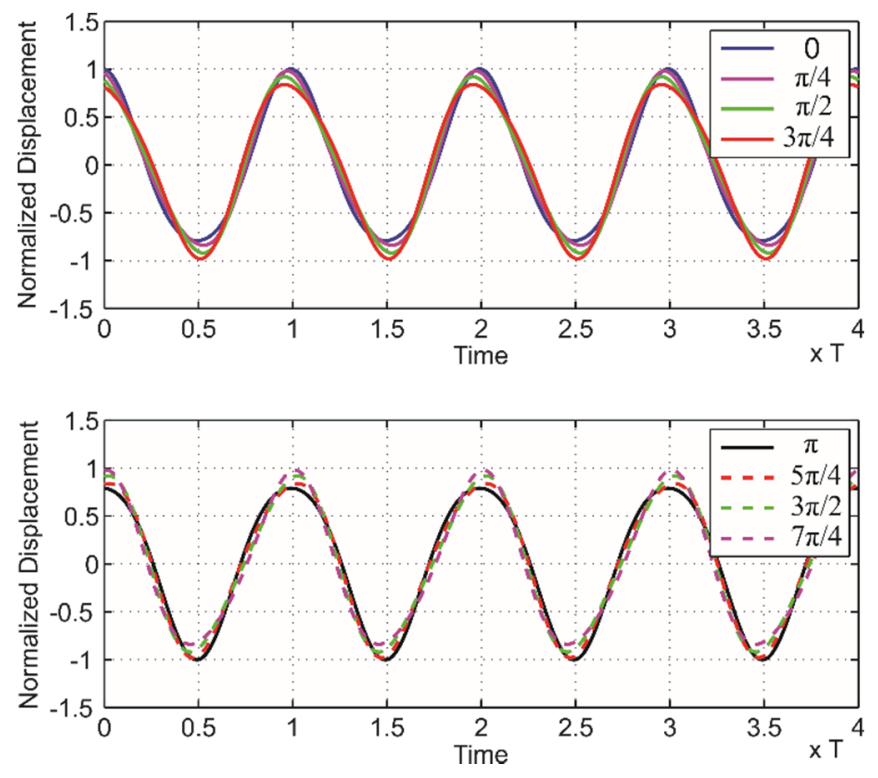

Fig.3 Normalized displacement of stator depending on phase difference $\varphi_{\mathrm{e}}$. High order harmonics at $2 \omega$ is integrated with fixed $D=0.3$ and $\mathrm{Qr}=0.2$.

\subsection{Design of the stator}

In this study, a bending stator with second harmonics at $2 \omega$ was designed to generate the saw-tooth type displacement driven by rectangular pulse waveform. It was composed of an aluminum plate sandwiched between two piezoelectric plates. The two PZT plates were identical and driven to produce opposite strain. As is known, the frequency ratio of the second and first bending resonance of a classical uniform beam with two free ends was approximately 2.76, so the length of PZT plates was designed to be half of the aluminum plate to generate step beam. 
Finite element analysis was conducted to optimize the dimensions of the stator using ATILA/GiD (Micromechatronics

Inc. Ver. 3.0.27) software. In the simulation, the length $\left(l_{a}\right)$ and thickness $\left(t_{a}\right)$ of the aluminum plate were $20 \mathrm{~mm}$ and $2 \mathrm{~mm}$, respectively. The length $\left(l_{p}\right)$ and thickness $\left(t_{p}\right)$ of the piezoelectric plates (PZT-8) were $10 \mathrm{~mm}$ and $0.73 \mathrm{~mm}$, respectively. The aluminum and piezoelectric plates had the same width $\left(w_{a}\right)$. The position of the PZT plates $\left(x_{p}\right)$ were optimized to obtain the required resonant frequency ratio, as shown in Fig.4.

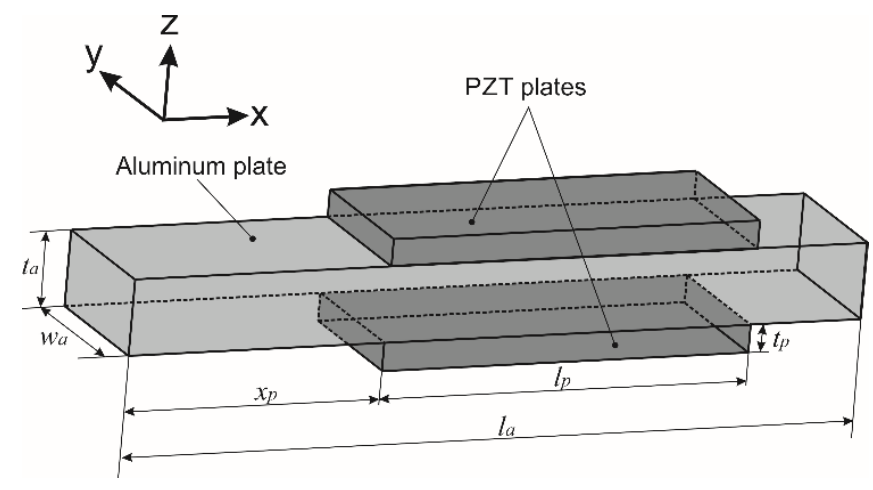

Fig.4 Structure of the stator composed by two symmetric piezoelectric plates and one aluminum plate

Fig. 5(a) shows the first $\left(\mathrm{f}_{1}\right)$ and second $\left(\mathrm{f}_{2}\right)$ bending resonant frequencies for various positions of PZT plates with the width of plates fixed to $4 \mathrm{~mm}$. The possibility of adjusting the ratio of the resonant frequencies to $1: 2$ with the position of PZT plates is clarified and the optimum position is estimated to be $6.235 \mathrm{~mm}$. It is shown in Fig.5(b) that the bending resonant frequencies keep stable with various plate widths. However, when the width gets close to $3 \mathrm{~mm}$, a bending mode in $\mathrm{x}-\mathrm{y}$ plane (where its resonant frequency is $\mathrm{f}_{\mathrm{c}}$ ) may also be excited because of fabrication and fixing errors, which affects the stator performance and should be avoided. Figs. 6 shows the first and second resonant bending modes in x-z plane, respectively. Theoretically, when the piezoelectric plates are placed in the center, second resonant mode cannot be excited. 


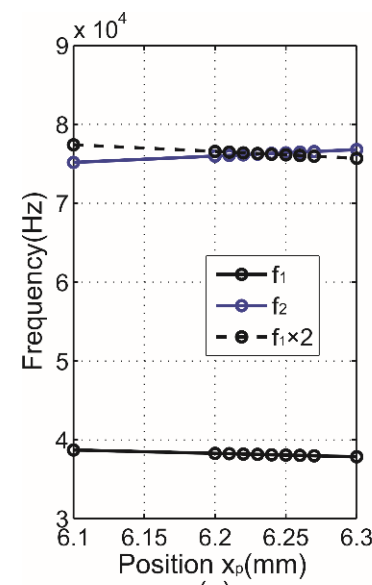

(a)

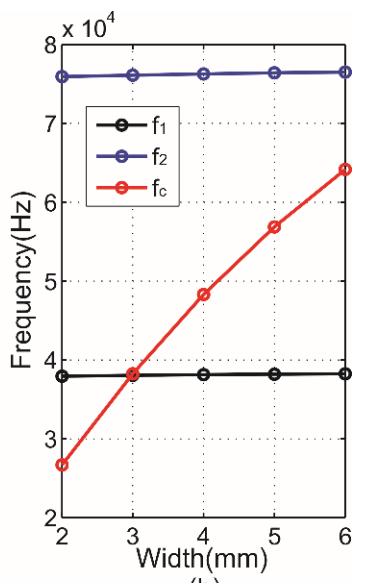

(b)

Fig.5 Resonant frequencies of bending modes for various (a) positions and (b) widths of PZT plates

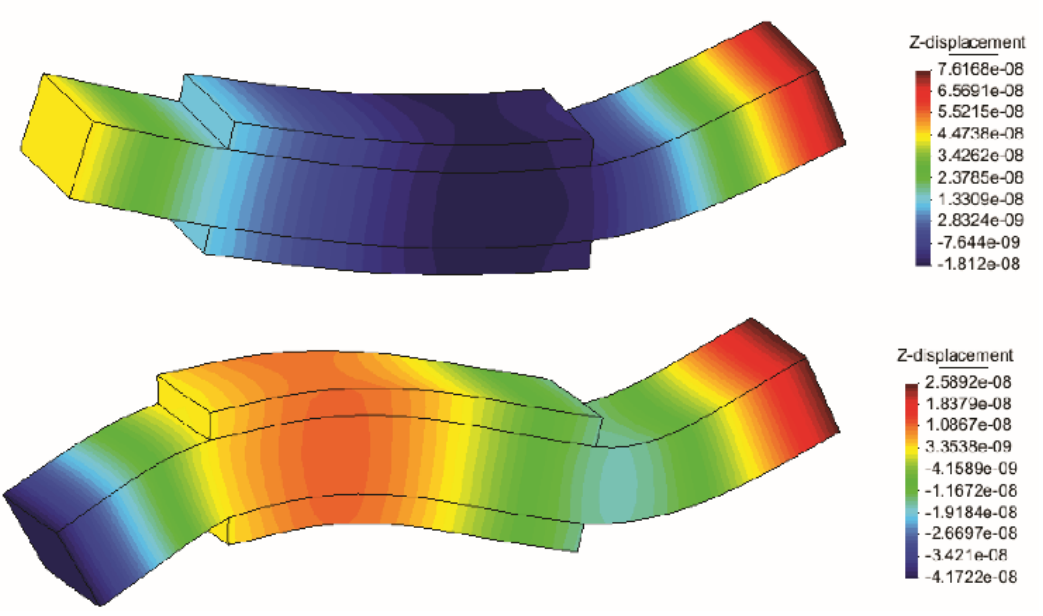

Fig.6 The fundamental and second bending resonant modes of the stator.

\section{Experiments}

\subsection{Experimental setup}


The stator was fabricated by two piezoelectric plates $(10 \mathrm{~mm} \times 4 \mathrm{~mm} \times 0.73 \mathrm{~mm})$ and one aluminum plate $(20 \mathrm{~mm} \times 4 \mathrm{~mm} \times 2 \mathrm{~mm})$. PZT-8 plates were supplied by Steiner and Martins Inc. and cut to a desired shape by an automatic dicing saw (DISCO DAD 3350). They were attached symmetrically to the aluminum plate with an epoxy adhesive (3M DP460). Because the PZT plates were both polarized in positive $z$ direction, the top and bottom electrodes were connected to the driving signal and the middle electrode (connected to the aluminum plate) was grounded. Though this stator can be adopted for both linear and rotary motors, we focus on the rotary motor characteristics in this paper.

The experimental setup and a prototype stator is shown in Fig.7. The stator is supposed to work at free-free boundary condition, so it was fixed on a base by a rubber sheet, to avoid restriction on its vibration. As the working mode combines both fundamental and second harmonics, the stator doesn't have any nodal points in this case. Therefore, according to the simulation results in Fig.6, the supporting point is located at $3.7 \mathrm{~mm}$ to the short end, which is the nodal point of second harmonic mode, in order to guarantee the production of saw-tooth type displacement. Then, the base was fixed on a precise positioning stage. The preload between stator and rotor was applied by the precise positioning stage and a spring that pressed on the supporting axis of the rotor. Rectangular pulse wave signals were generated by a function generator (SIGLENT SDG1025) and amplified by a power amplifier (NF 4010). A Laser Doppler Vibrometer (PolyTec OFV 3001/OFV 511) was utilized to measure the vibration velocity of the stator. To measure the angular speed of the bearing rotor, a code wheel was used. Also, photoelectric encoder was used to detect the rotation pulse generated by the code wheel. In addition, a Labview program was compiled to read the pulse and 
calculate the angular speed. The stall torque was tested using a mass and electronic scale and it could be evaluated by the maximum mass the rotor could lift.

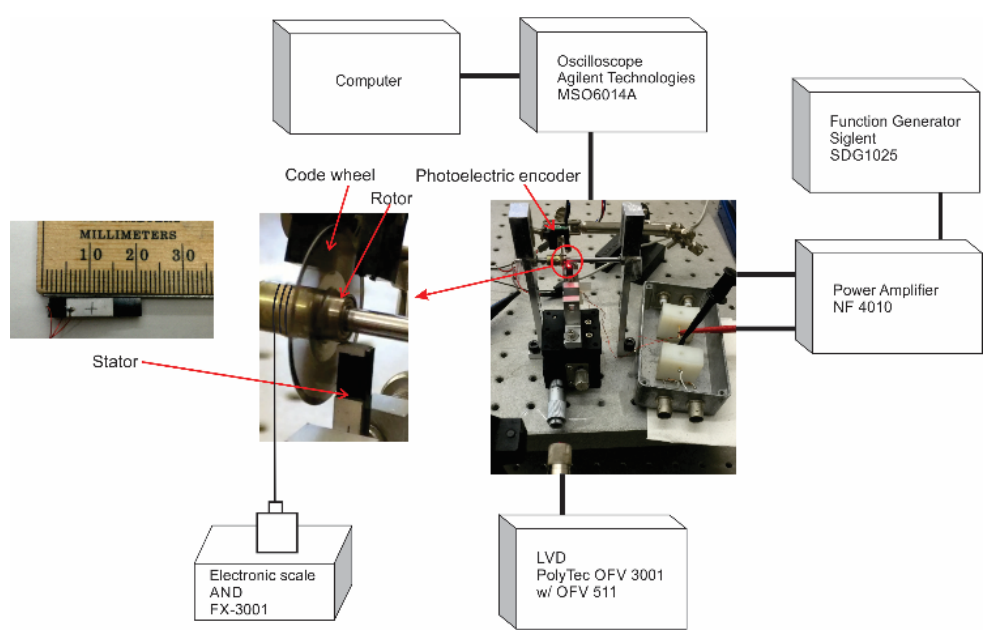

Fig.7 Experimental setup for motor performance evaluation

\subsection{Tests and results}

\subsubsection{Characteristics of the stator}

The impedance spectrum of the stator measured by Agilent 4294A is shown in Fig. 8. Because the phase angle could not exhibit zero (which corresponds to the theoretical resonance frequency), the maximum phase points were chosen as they were quiet close to the points with maximum vibration velocity, which were $70390 \mathrm{~Hz}$ and $35240 \mathrm{~Hz}$, respectively, with the frequency ratio being 1.997 , which basically satisfies the $2: 1$ ratio requirement. 


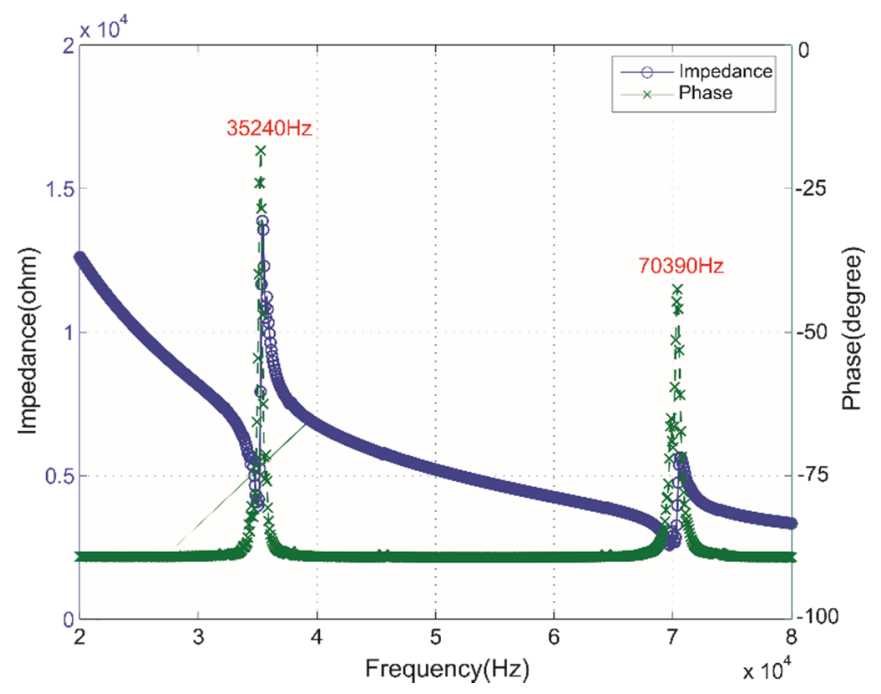

Fig.8 Impedance curve of the stator
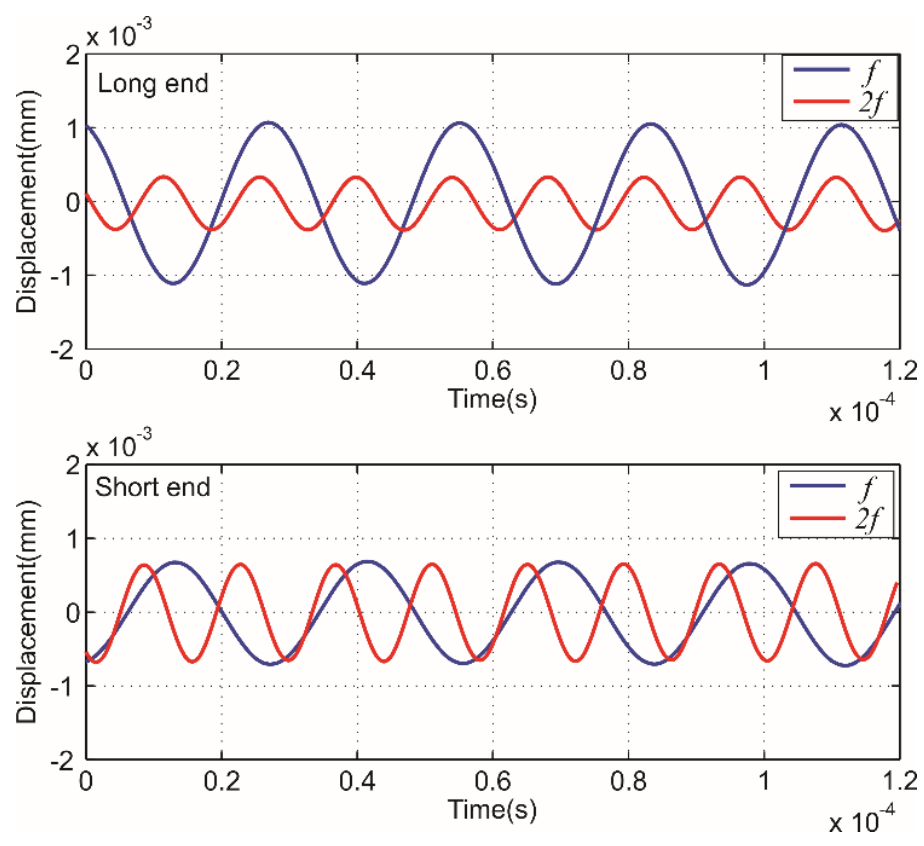

Fig. 9 Displacement of two free ends of the stator driven by sinusoidal waveform at $35300 \mathrm{~Hz}$ and $70600 \mathrm{~Hz}$ 

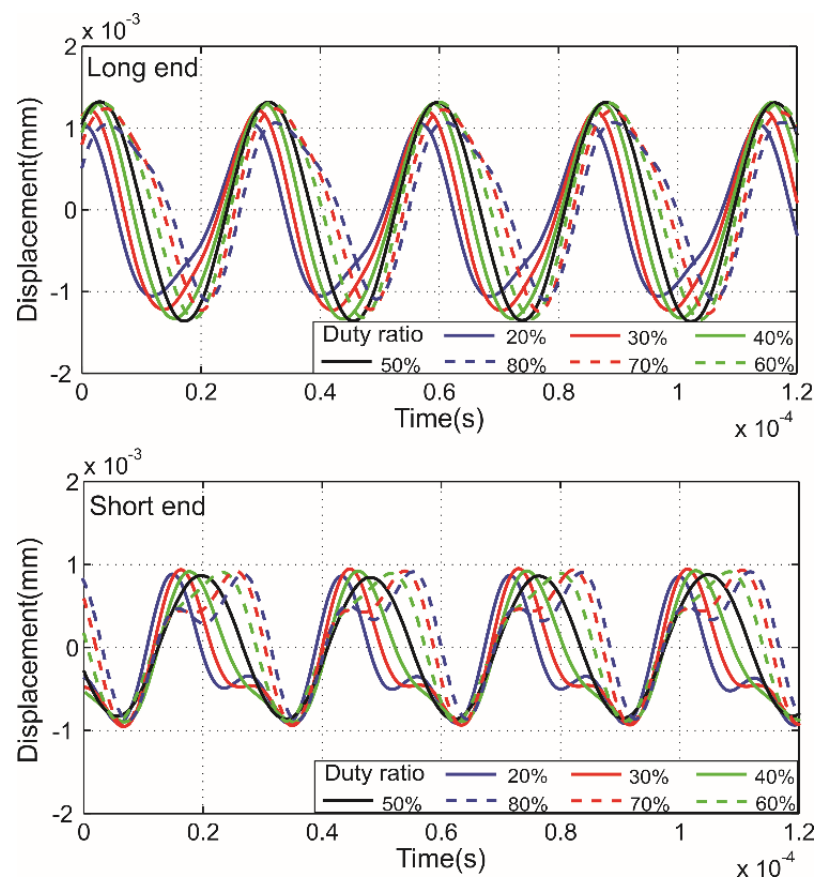

Fig.10 Displacements of two free ends of the stator driven by pulse waveform with different duty ratio

As the piezoelectric plates were not placed at the center of the aluminum plate, the displacements of the two free ends of the stator were different when the stator was driven by sinusoidal signal at fundamental $(35300 \mathrm{~Hz})$ and double fundamental frequencies $(70600 \mathrm{~Hz})$. The long end was defined as the free end with a longer aluminum plate and the other end was shorter. As shown in Fig.9, the long end produced a larger vibration amplitude at fundamental frequency, while the short end presented a larger vibration amplitude at double fundamental frequency, under driving voltage amplitude of 40Vpp. The Qr and $\varphi$ e for the long end was approximately 0.33 and $1.68 \pi$, while for the short end, the Qr and $\varphi$ e was approximately 0.96 and $0.17 \pi$. The measured displacements of the stator driven by rectangular pulse are shown in Fig. 10. Both ends of the stator could be used to drive the rotor because the saw-tooth type displacements could be generated on both ends. According to analysis in Section 2, when $n=2$, Qr is small(less than 0.8), the 
displacement curve becomes more asymmetric and the vibration amplitude decreases as the duty ratio deviates from $50 \%$. When $\varphi$ e is $1.68 \pi$, which is between $\pi$ and $2 \pi$, the displacement curve will lean to the right side when duty ratio is less than $50 \%$. When $\varphi$ e is $0.17 \pi$, which is between 0 and $\pi$, the displacement curve will lean to the left side when duty ratio is less than $50 \%$. When Qr is 0.33 , the displacement curve will present saw-tooth type curve with one peak, while when Qr is 0.96 (larger than 0.8), the displacement curve will present two peak shape. These features are all in accordance with previous analysis.

The influence of the high order harmonic is basically determined by its vibration amplitude and frequency. The resonant frequencies of fundamental, second and third harmonics of the prototype are $35.3 \mathrm{kHz}, 70.6 \mathrm{kHz}$ and $118.2 \mathrm{kHz}$. The third harmonic shows large deviation $(11 \%)$ from $3 \omega(35.3 \times 3=105.9 \mathrm{kHz})$ and even larger deviation $(16 \%)$ from $4 \omega(35.3 \times 4=141.2 \mathrm{kHz})$. So finally, the ratio of vibration amplitude of the stator (long end) driven by the same voltage at $\omega, 2 \omega, 3 \omega$ is 1:0.33:0.009. For rectangular drive, $a_{n}$ and $b_{n}$ are proportional to $1 / n$, so the vibration amplitude will be further reduced for third and even higher harmonic mode and their influence on the output can be neglected.

Since the long end presented a larger vibration amplitude with the same driving voltage, the rotor was driven at the long end in the following experiments.

\subsubsection{Motor performance}

Two kinds of rotors were used for the motor performance test. One was a light-weight rotor with a mass of $0.543 \mathrm{~g}$ and a preload of $0.05 \mathrm{~N}$ was applied. The other was a large rotor with a mass of $108.7 \mathrm{~g}$ and a preload of $1.07 \mathrm{~N}$ was applied. 
The frequency shifting should be considered when the large rotor is used. The relationship between the angular speed of the motor and the exciting frequency is shown in Fig. 11. When the rectangular pulse driving voltage was set to $70 V_{\mathrm{pp}}$ with a duty ratio of $30 \%$, the rotor speed reached maximum of $45.8 \mathrm{rpm}$ at frequency of $35.2 \mathrm{kHz}$. As the optimum driving frequency only presented $0.3 \%$ reduction, driving frequency of $35.3 \mathrm{kHz}$ was used in the following experiments.

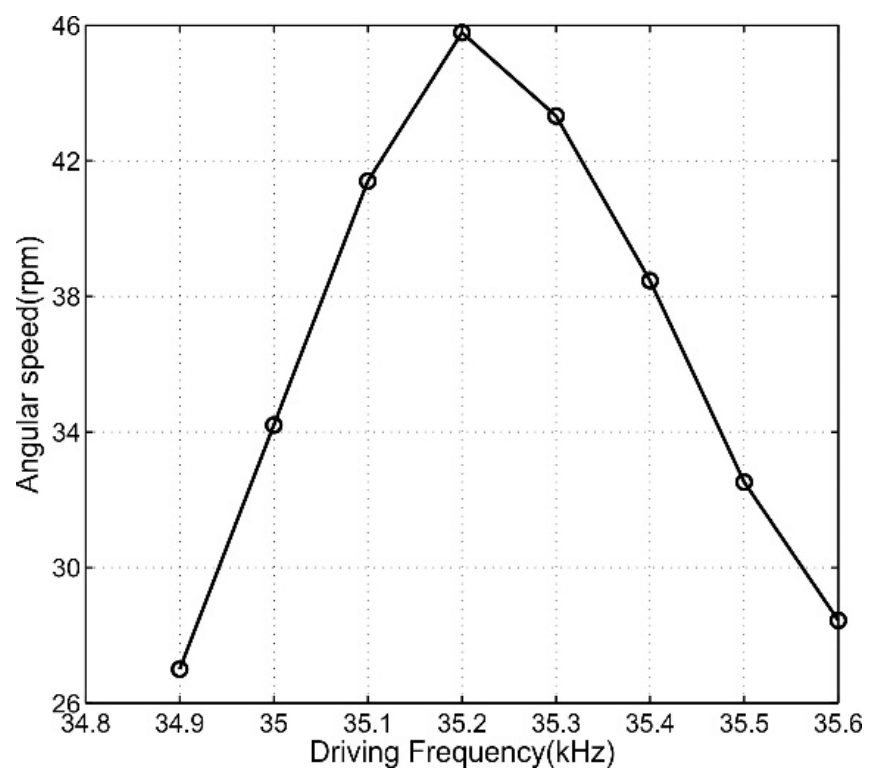

Fig.11 Angular speed versus driving frequency with the large rotor

Figures 12-16 show the performances of the motor with two rotors. The angular speed of the rotor was set to be negative when the rotor was driven by duty ratio of higher than $50 \%$, meaning the rotor rotates in a reverse direction.

Figure 12 shows the relationship between angular speed and driving voltage amplitude, with duty ratio of $30 \%$ (positive direction) and $70 \%$ (negative direction). The driving frequency was set to $35.3 \mathrm{kHz}$. For the small rotor, the angular speed was linear to the input voltage ranging from $30 \mathrm{Vpp}$ to $60 \mathrm{Vpp}$ and reached $295 \mathrm{rpm}$ and $298 \mathrm{rpm}$ in each direction with a driving voltage of $60 \mathrm{Vpp}$. For the larger rotor, the angular speed was about $83 \%$ reduction compared 
with small rotor. It should be noted that in order to get the best performance in both directions, the contact position of the stator and rotor were slightly changed when the performance in each direction was measured.

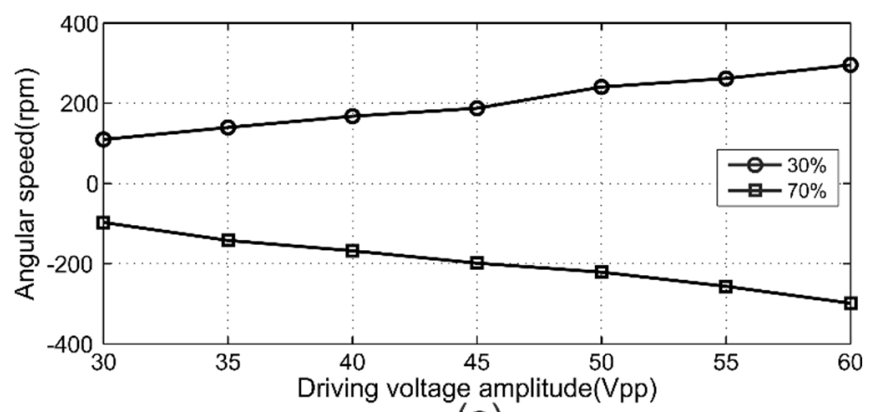

(a)

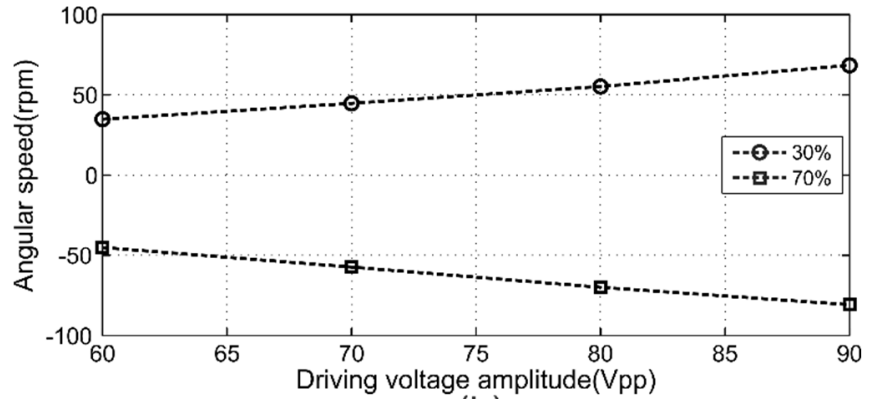

(b)

Fig.12 Angular speed versus driving voltage amplitude in both directions using (a) small rotor and (b) large

rotor.

Figure 13 shows the curve of the angular speed versus the applied voltage's duty ratio with different driving voltage

at frequency of $35.3 \mathrm{kHz}$. Because the driving voltage amplitude was significantly attenuated, the rotor did not rotate with duty ratio close to $0 \%$ and $100 \%$. The rotor also did not move with duty ratio near $50 \%$ because no obvious sawtooth type displacement was output. For the small rotor, the angular speed reached a peak value with the duty ratio in between $35 \%-45 \%$ and $60 \%-70 \%$ in two directions. In order to simplify the electronic circuit, duty ratio of 35\%-40\% $(D)$ and $60 \%-65 \%(1-D)$ could be used in practical applications. For the larger rotor, the angular speed peaked at duty 
ratio of $30 \%-40 \%$ and $60 \%-70 \%$ in two directions. According to the previous analysis, as the duty ratio deviates from $50 \%$, the displacement curve becomes more asymmetric, but the vibration amplitude decreases, so there is an optimal value of duty ratio to generate the highest speed.

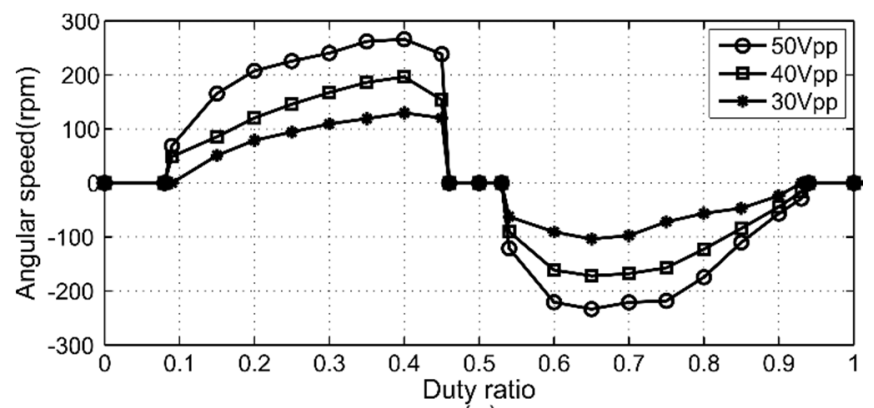

(a)

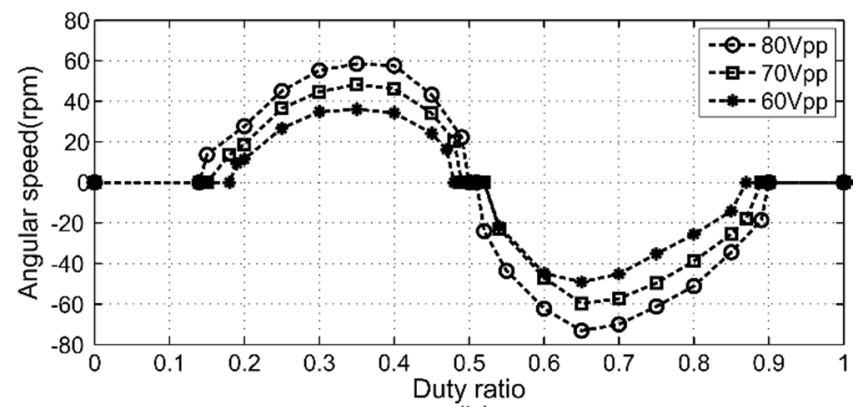

(b)

Fig.13 Angular speed versus duty ratio with different driving amplitude using (a) small rotor and (b) large

rotor.

Figure 14 shows the relationship between stall torque and driving voltage amplitude in both directions. The driving

frequency was set to $35.3 \mathrm{kHz}$. The stall torque increased with the driving voltage amplitude and became saturated afterward. For the large motor, the stall torque remained almost at $1161 \mu \mathrm{Nm}$ when the driving amplitude kept increasing after $80 \mathrm{Vpp}$ in negative direction. Similarly, the increase of stall torque gradually slowed down until it got almost saturation at $80 \mathrm{Vpp}$, with stall torque of $1220 \mu \mathrm{Nm}$ in positive direction. The torque of small rotor is much smaller, about $90 \%$ reduction compared with larger rotor. 
The acceleration provided by friction force is defined as a (for simplicity, the stick and slip friction coefficients are assumed to be equal), and the acceleration of bending actuator is defined as $a b$. If $a b<a 0$, the rotor will stick with the stator throughout the whole working period, which is called stick-stick motion. In this case, the motor will not work. If $\mathrm{a}_{\mathrm{b}}>\mathrm{a}_{0}$, the motor will work in several stick-slip motions, and the acceleration of rotor in "stick" motion is determined by the acceleration of vibrating stator ab, while the rotor acceleration in "slip" motion depends on friction acceleration $\mathrm{a}_{0}[13-15]$. With the increase of vibration acceleration, the "stick" motion will be less and the performance of the rotor is basically determined by friction force. As the preload and material is fixed during operation, both the rotation speed and stall torque will become saturated eventually even the driving amplitude increases continuously.

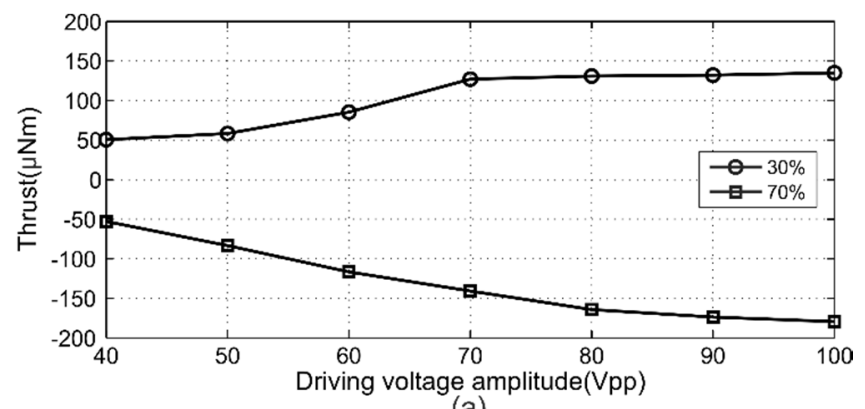

(a)

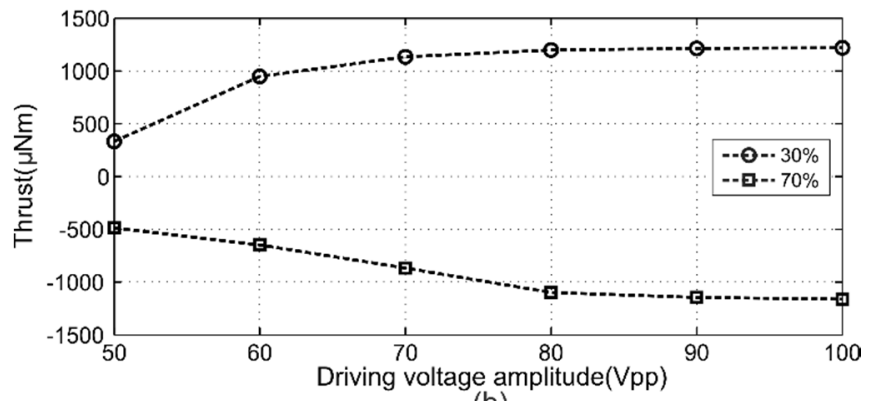

(b)

Fig.14 Stall torque versus driving voltage amplitude in both directions using (a) small rotor and (b) large 
Figure 15 shows the curve of the stall torque versus the applied voltage's duty ratio when the driving voltage was $60 \mathrm{Vpp}$ and $35.3 \mathrm{kHz}$. For the large rotor, the stall torque reached a peak value in between duty ratio of $25 \%-35 \%$ and $65 \%-75 \%$ in two directions. For the small rotor, the torque is much smaller, about $90 \%$ reduction compared with larger rotor, and the duty ratio of 30\%-35\% and 65\%-70\% could be used in practical applications.

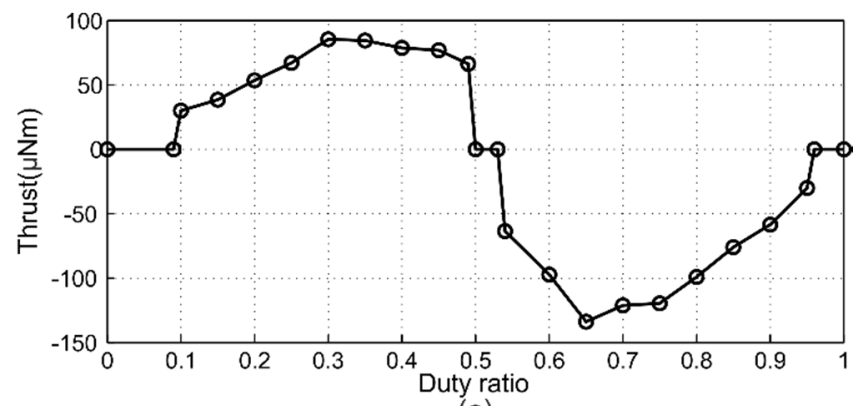

(a)

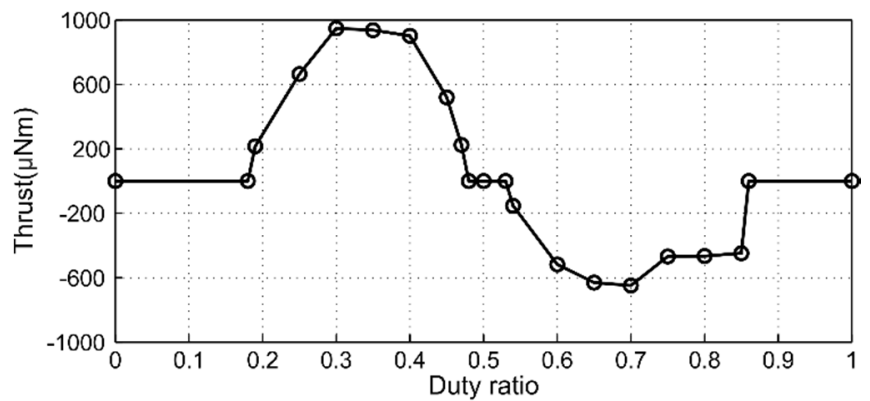

(b)

Fig. 15 Stall torque versus duty ratio with driving amplitude of $60 \mathrm{Vpp}$ at $35.3 \mathrm{kHz}$ using (a) small rotor and (b) large rotor

The angular speed and efficiency as a function of the load is shown in Fig. 16. The driving amplitude and frequency were set to $55 \mathrm{Vpp}$ and $35.3 \mathrm{kHz}$, with a duty ratio of $30 \%$. For the small rotor, the motor could rotate at a speed of 176rpm with a load of $7.2 \mu \mathrm{Nm}$ and output torque of $70.4 \mu \mathrm{Nm}$ when maintaining a rotational speed of $25.2 \mathrm{rpm}$. The maximum efficiency was about $2 \%$ with a load of approximately $39.3 \mu \mathrm{Nm}$. The large rotor presented a larger torque and lower speed, with almost the same maximum efficiency. 


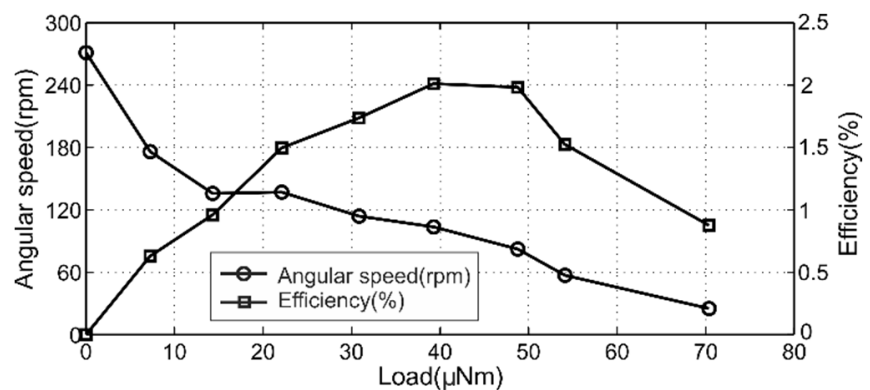

(a)

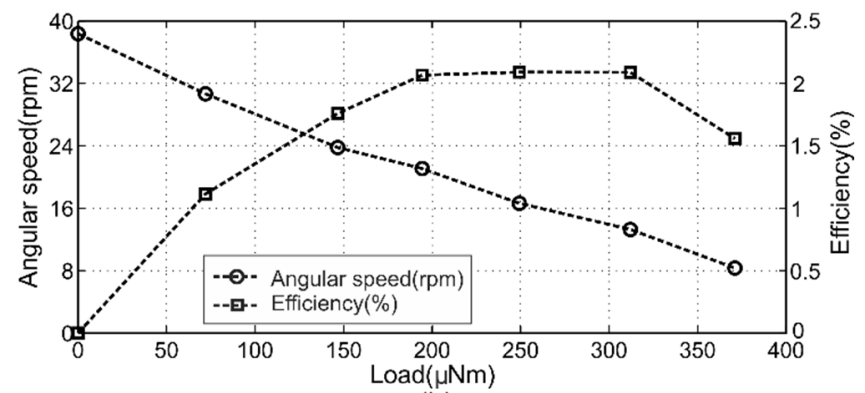

(b)

Fig.16 Angular speed and motor efficiency versus load using (a) small rotor and (b) large rotor, with driving voltage setting at $55 \mathrm{Vpp}, 35.3 \mathrm{kHz}$, with a duty ratio of $30 \%$.

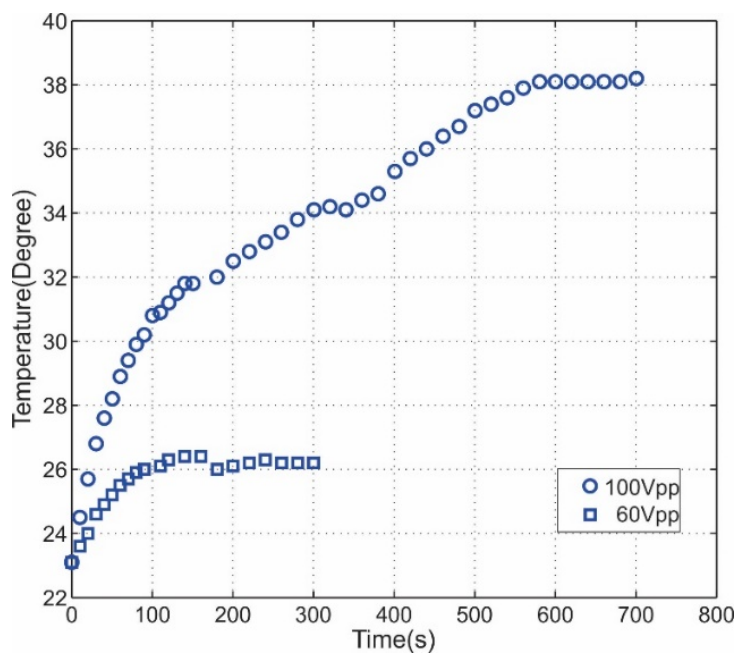

Fig.17 Temperature rising of the stator with driving amplitude of 60Vpp and 100Vpp. 
In order to insure the effective work of the motor, the heat generation of the stator should be considered to avoid invalidation of epoxy. The temperature rise of the stator was measured and shown in Fig.17. The room temperature was around $23^{\circ} \mathrm{C}$. When the stator was driven by a voltage of $60 \mathrm{Vpp}$, the temperature stayed at $26^{\circ} \mathrm{C}$ after a continuous running of 100 seconds. Even if the stator was driven by a voltage of $100 \mathrm{Vpp}$, the temperature of the stator rose by $15^{\circ} \mathrm{C}$ after continually running for 10 minutes, then remaining stable afterwards. Therefore, the motor could work continuously without much heat generation.

\subsection{Comparison with state-of-art}

Table 1 compares the performance of the proposed inertial motor with its counterparts reported before. The efficiency of the motor is considerably increased compared with static type and the power density is also larger. A small rotor can be used for high rotation applications with $5 \mathrm{rpm} / \mathrm{Vpp}$, which is larger than most previous inertial motors. On the other hand, a large rotor could be used for high torque applications with $15 \mu \mathrm{Nm} / \mathrm{Vpp}$. Although this value is smaller than that of static motor reported by Morita in 1999, the structure of the stator is much simpler, which is one of the primary advantages of inertial motors.

Table 1 Performance comparison with previously reported piezoelectric inertial rotary motors

\begin{tabular}{|l|l|l|l|l|l|l|l|}
\hline Author(year) & Type & Size of & Frequency & Angular speed & Stall & Max. & Max. Power \\
& & stator(mm & $(\mathrm{kHz})$ & $(\mathrm{rpm} / \mathrm{Vpp})$ & torque $(\mu \mathrm{Nm} /$ & Efficiency & Density \\
& & & & & & Vpp $)$ & \\
\hline
\end{tabular}




\begin{tabular}{|c|c|c|c|c|c|c|c|}
\hline T. Morita(1999)[5] & static & $\varnothing 15 \times 11$ & 8 & $27 / 180$ & $5488 / 180$ & $0.045 \%$ & 2851 \\
\hline W. X. Han(2009)[16] & static & $\varnothing 1 \times 15$ & 8 & $90 / 600$ & $80 / 1000$ & --- & --- \\
\hline Q. Zhang(2012)[17] & static & $\varnothing 8 \times 10$ & 8 & $22.5 / 1400$ & $1600 / 1400$ & $0.1 \%$ & 3190 \\
\hline S. & resonant & $\varnothing 5 \times 25$ & 34 & $29 / 20$ & --- & --- & --- \\
\hline T. Morita(2012)[12] & resonant & $10 \times 2 \times 0.7$ & 290 & $400 / 80$ & --- & --- & --- \\
\hline Our motor & resonant & $20 \times 4 \times 3.4$ & 35.3 & $300 / 60$ & $1198 / 80$ & $2.1 \%$ & 4156 \\
\hline
\end{tabular}

\section{Conclusion}

An rectangular pulse drive for resonant-type piezoelectric inertial motor is explained and a rotary motor with

bending stator is developed to verify its effectiveness. Basically, high order harmonics at even multiple fundamental

resonant frequencies without large amplitude attenuation will benefit the construction of saw-tooth type displacement

of the stator. A prototype stator was fabricated with fundamental and second bending resonant frequencies of $35300 \mathrm{~Hz}$ and $70600 \mathrm{~Hz}$ and its performances were evaluated by driving two rotors. The moving direction of the rotor could be easily reversed by changing the duty ratio. With optimized duty ratios, both the rotation speed and stall torque could be enhanced. In addition to larger power density, the efficiency of the prototype motor is considerably increased compared with static type inertia motor. The small rotor can be used for high rotation applications with $5 \mathrm{rpm} / \mathrm{Vpp}$, while a large preload could be used for high torque applications with $15 \mu \mathrm{Nm} / \mathrm{Vpp}$. Because of the simple structure 
and low cost of both mechanical and electrical parts, the proposed motor is a promising devices for use in camera,

robots and medical equipment.

\section{Acknowledgment}

Part of this work at the Penn State University is supported by the Office of Naval Research under grant number: ONR

N00014-14-1-1044. One of the authors, Yuting Ma was also supported by the China Scholarship Council (CSC). This

work is also supported by National Natural Science Foundation of China: Project 51305439.

\section{Reference}

[1] M. Anders, M. Thaer, C. Heiden, Simple micropositioning devices for STM, Sur-face Science 181 (1987) 176182.

[2] D.W. Pohl, Dynamic piezoelectric translation devices, Review of Scientific Instruments 58 (1987) 54-57.

[3] Ph. Niedermann, R. Emch, P. Descouts, Simple piezoelectric translation device, Rev. Sci. Instrum. 59 (1988) 368-369.

[4] Higuchi T, Yamagata Y, Furutani K, et al. Precise positioning mechanism utilizing rapid deformations of piezoelectric elements[C]. Proceedings. IEEE Micro Electro Mechanical Systems-An Investigation of Micro Structures, Sensors, Actuators, Machines, 1990: 222-226.

[5] Takeshi Morita, Ryuichi Yoshida, Yasuhiro Okamoto et al. A smooth impact rotation motor using a multilayered torsional piezoelectric autuator[C]. IEEE Transactions on Ultrasonics, Ferroelectrics and Frequency Control, 1999, 46(6): 655-663.

[6] K.J. Lim, J.S. Lee, S.H. Park, S.H. Kang, H.H. Kim, Fabrication and characteristics of impact type ultrasonic motor, J. Eur. Ceram. Soc. 27 (13) (2007)4159-4162.

[7] D. Paik, K. Yoo, C. Kang, B. Cho, S. Nam, S. Yoon, Multilayer piezoelectric linear ultrasonic motor for camera module, Journal of Electroceramics 22 (2009)346-351.

[8] J. Lee, W.S. Kwon, K. Kim, S. Kim, A novel smooth impact drive mechanism actuation method with dual-slider for a compact zoom lens system, Review of Scientific Instruments 82 (2011) 085105.

[9] S. Tuncdemir, S.O. Ural, B. Koc, K. Uchino, Design of translation rotary ultrasonicmotor with slanted piezoelectric ceramics, Japanese Journal of Applied Physics50 (2011) 027301.

[10] R. Bansevicius, V. Blechertas, Multi-degree-of-freedom ultrasonic motors for mass-consumer devices, Journal of Electroceramics 20 (2008) 221-224.

[11] T. Nishimura, H. Hosaka, T. Morita, Resonant-type smooth impact drive mechanism (SIDM) actuator using a bolt-clamped langevin transducer, Ultrasonics52 (2012) 75-80. 
[12] T. Morita, H. Murakami, T. Yokose, H. Hosaka, A miniaturized resonant-type smooth impact drive mechanism actuator, Sensors and Actuators A: Physical178 (2012) 188-192.

[13] Qiao Sheng Pan, Liang Guo He, Cheng Liang Pan, Guang Jun Xiao, Zhi Hua Feng, Resonant-type inertia linear motor based on the harmonic vibration synthesis of piezoelectric bending actuator, Sensors and Actuators A 209

(2014) 169-174.

[14] M. Hunstig, T. Hemsel, W. Sextro, Stick-slip and slip-slip operation of piezo-electric inertia drives. Part I: ideal excitation, Sensors Actuat. A Phys. 200 (2013)90-100.

[15] M. Hunstig, T. Hemsel, W. Sextro, Stick-slip and slip-slip operation of piezo-electric inertia drives. Part II: frequency-limited excitation, Sensors Actuat. APhys. 200 (2013) 79-89.

[16] W. X. Han, Q. Zhang, Y. T. Ma, C. L. Pan, and Z. H. Feng, "An impact rotary motor based on a fiber torsional piezoelectric actuator,” Rev. Sci. Instrum., vol. 80, no. 1, p. 014701, 2009.

[17] Zhang Qi, Pan Cheng Liang, Ma Yu Ting, Kong Fan Rang, and Feng Zhi Hua, Piezoelectric Rotary Motor Based on Active Bulk Torsional Element With Grooved Helical Electrodes, IEEE/ASME Transactions on mechatronics, Vol. 17, No. 2, 2012. 
Yuting Ma was born in 1984. She received her $\mathrm{Ph}$. D. degree from the university of Science and Technology of China, Hefei, China, in 2011. Currently, she is an associate professor in CAS Key Lab of Bio-Medical Diagnostics, Suzhou Institute of Biomedical Engineering and Technology, Chinese Academy of Sciences, Suzhou, Jiangsu, China. In 2015-2016, she works at the International Center for Actuators and Transducers, The Pennsylvania State University as a visiting scholar. Her research interests include piezoelectric actuators and their applications, transducers and medical instruments.

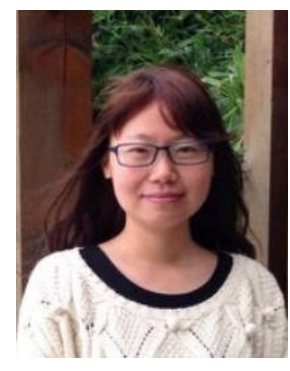

Husain N. Shekhani was born in Karachi, Pakistan on October 18, 1989. He received his B.S. degree in mechanical engineering from the Missouri University of Science and Technology in 2012, during which time he memorized the Holy Quran. After this, he joined the Pennsylvania State University in Dr. K. Uchino's research group, the International Center for Actuators and Transducers. The subject of his research is mainly directed toward development of new methodologies for measuring piezoelectric material properties in high power conditions. He has published several journal articles on piezoelectric material property characterization. Following his spirit to share his experiences, in 2015 founded the first online platform dedicated to teach basic knowledge regarding piezoelectric materials via free online video lectures: LearnPiezo.org.

Xintao Yan was born in 1987. He received his M.E. degree from Harbin institute of technology in 2011. He is now a research assistant in CAS Key Lab of Bio-Medical Diagnostics, Suzhou Institute of Biomedical Engineering and Technology, Chinese Academy of Sciences, Suzhou, Jiangsu, China. His research focuses on mechanical design and dynamic modeling.

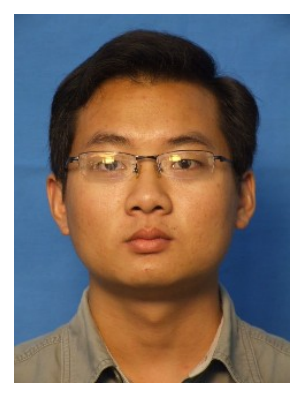

Minkyu Choi received the B.S. and M.S. degrees in materials science and engineering from Korea University, Seoul, South Korea in 2007 and 2009, respectively. He contributed to develop dispenser head systems in Protec and 6-axis inertial MEMS sensors in Samsung Electro-Mechanics as a researcher. He is 
currently a Ph.D. candidate in International Center for Actuators and Transducers. His research interests cover the design and analysis of piezoelectric materials, losses, and its applications.

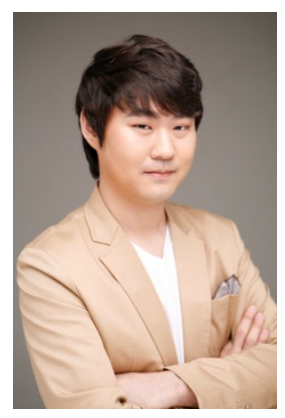

Kenji Uchino is Founding Director of International Center for Actuators and Transducers and Professor of EE and MatSE at Penn State University since 1991. He was Associate Director at The US Office of Naval Research - Global Tokyo Office, and also the Founder and Senior Vice President of Micromechatronics Inc., State College, PA. He was also awarded his MBA degree in 2008, and authored a textbook 'Entrepreneurship for Engineers'. He is a Fellow of American Ceramic Society and of IEEE, and also is a recipient of 28 awards, including International Ceramic Award from Global Academy of Ceramics (2016), IEEE-UFFC Ferroelectrics Recognition Award (2013).

His research interest is in solid state physics, especially in ferroelectrics and piezoelectrics, including basic research on theory, materials, device designing and fabrication processes, as well as application development of solid state actuators/sensors for precision positioners, micro-robotics, ultrasonic motors, smart structures, piezoelectric transformers and energy harvesting. He has authored 520 papers, 68 books and 31 patents in the ceramic actuator area.

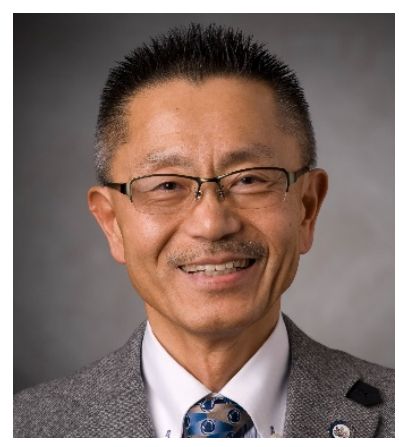


Table 1 Performance comparison with previously reported piezoelectric inertial rotary motors

\begin{tabular}{|c|c|c|c|c|c|c|c|}
\hline Author(year) & Type & $\begin{array}{l}\text { Size of } \\
\text { stator(mm } \\
\text { ) }\end{array}$ & $\begin{array}{l}\text { Frequency } \\
(\mathrm{kHz})\end{array}$ & $\begin{array}{l}\text { Angular speed } \\
\text { (rpm/Vpp) }\end{array}$ & $\begin{array}{l}\text { Stall } \\
\text { torque }(\mu \mathrm{Nm} / \\
\text { Vpp) }\end{array}$ & $\begin{array}{l}\text { Max. } \\
\text { Efficiency }\end{array}$ & $\begin{array}{l}\text { Max. Power } \\
\text { Density } \\
\left(\mathrm{W} / \mathrm{m}^{3}\right)\end{array}$ \\
\hline T. Morita(1999)[5] & static & $\varnothing 15 \times 11$ & 8 & $27 / 180$ & $5488 / 180$ & $0.045 \%$ & 2851 \\
\hline W. X. Han(2009)[16] & static & $\varnothing 1 \times 15$ & 8 & $90 / 600$ & $80 / 1000$ & --- & --- \\
\hline Q. Zhang(2012)[17] & static & $\varnothing 8 \times 10$ & 8 & $22.5 / 1400$ & $1600 / 1400$ & $0.1 \%$ & 3190 \\
\hline $\begin{array}{l}\text { S. } \\
\text { Tuncdemir(2011)[9] }\end{array}$ & resonant & $\varnothing 5 \times 25$ & 34 & $29 / 20$ & --- & --- & --- \\
\hline T. Morita(2012)[12] & resonant & $10 \times 2 \times 0.7$ & 290 & $400 / 80$ & --- & --- & --- \\
\hline Our motor & resonant & $20 \times 4 \times 3.4$ & 35.3 & $300 / 60$ & $1198 / 80$ & $2.1 \%$ & 4156 \\
\hline
\end{tabular}

